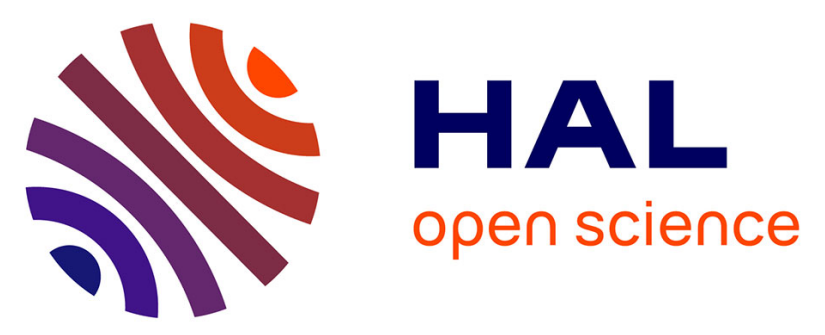

\title{
Carrier Aggregation/Channel Bonding in Next Generation Cellular Networks: Methods and Challenges
}

Zaheer Khan, Hamed Ahmadi, Ekram Hossain, Marceau Coupechoux, Luiz Dasilva, Janne Lehtomäki

\section{- To cite this version:}

Zaheer Khan, Hamed Ahmadi, Ekram Hossain, Marceau Coupechoux, Luiz Dasilva, et al.. Carrier Aggregation/Channel Bonding in Next Generation Cellular Networks: Methods and Challenges. IEEE Network, 2014, 28 (6), pp.34-40. 10.1109/MNET.2014.6963802 . hal-01109630

\section{HAL Id: hal-01109630 \\ https://hal-imt.archives-ouvertes.fr/hal-01109630}

Submitted on 24 Oct 2015

HAL is a multi-disciplinary open access archive for the deposit and dissemination of scientific research documents, whether they are published or not. The documents may come from teaching and research institutions in France or abroad, or from public or private research centers.
L'archive ouverte pluridisciplinaire HAL, est destinée au dépôt et à la diffusion de documents scientifiques de niveau recherche, publiés ou non, émanant des établissements d'enseignement et de recherche français ou étrangers, des laboratoires publics ou privés. 


\title{
Carrier Aggregation/Channel Bonding in Next
}

\section{Generation Cellular Networks: Methods and Challenges}

\author{
Zaheer Khan, Hamed Ahmadi, Ekram Hossain, Marceau Coupechoux, Luiz A DaSilva, \\ and Janne J. Lehtomäki
}

\begin{abstract}
To provide cellular systems with additional spectral resources, the wireless industry is considering the aggregation of frequency carriers in licensed, unlicensed, and shared access (SA) bands. In this article, we focus on reliable carrier aggregation/channel bonding $(\mathrm{CA} / \mathrm{CB})$ techniques, in which when $\mathrm{CA} / \mathrm{CB}$ between the licensed, unlicensed and SA carriers is performed, the licensed carrier is used for the primary and secondary carriers, and the unlicensed and SA carriers are operated as additional secondary carriers. We provide a taxonomy of the use of $\mathrm{CA} / \mathrm{CB}$ in cellular networks and highlight the differences between different $\mathrm{CA} / \mathrm{CB}$ approaches. We make the case that although the licensed primary carrier can give reliable transmission of control signalling, due to the nature of unlicensed and SA bands, for the efficient aggregation of secondary carriers there is a need for new CA/CB methods. To illustrate our case for novel CA/CB methods, we provide examples for different network environments where intelligent $\mathrm{CA} / \mathrm{CB}$ decisions can increase throughput as compared to the traditional CA/CB methods. Finally, we highlight challenges in the design of novel CA/CB techniques in unlicensed and SA bands.
\end{abstract}

\section{Index Terms}

Authorized shared access; carrier aggregation; channel bonding; heterogeneous networks; licensed access; opportunistic access; shared access; unlicensed access.

\section{INTRODUCTION}

To address the so-called 1000x mobile data challenge, researchers have started investigating the next generation of cellular networks. Compared to $4 \mathrm{G}$ networks, the next generation of cellular networks are envisioned to achieve 1000 times the system capacity [1], [2]. Technological innovations such as mmWave communication and visible light communication (VLC) can offer very high data rates; however, these are disruptive technologies and for deployment they will require huge economic resources and radical changes

Z. Khan and Janne J. Lehtomäki are with University of Oulu, Finland; Hamed Ahmadi and L. A. DaSilva are with Telecommunications Research Centre, Ireland; E. Hossain is with University of Manitoba, Canada; and Marceau Coupechoux is with Telecom Paris Tech, France.

This work was funded by Academy of Finland, National Science Foundation, USA, Natural Sciences and Engineering Research Council of Canada (NSERC), and Science Foundation Ireland. 
to wireless systems at both the component and architecture levels. Techniques that can aggregate carriers simultaneously across different spectrum bands such as licensed, unlicensed, and shared access (SA) bands can also offer increased user data rates [3]. Moreover, unlike the disruptive technologies mentioned above, they enable devices to co-exist seamlessly with existing wireless systems. In wireless networks, aggregation of carriers is enabled by carrier aggregation (CA) techniques and channel bonding $(\mathrm{CB})$ techniques. $\mathrm{CA}$ techniques can aggregate multiple contiguous and non-contiguous carriers (in different spectrum bands) to be used together [3]. In wireless networks, such as WiFi networks, CB techniques are used to combine multiple adjacent channels to form larger transmission bandwidths [4], [5]. CB approaches can be across different unlicensed spectrum bands, such as $2.4 \mathrm{GHz}$ and $5 \mathrm{GHz}$ bands.

It is worth noting that CA can also be leveraged in the context of millimeter wave (mmW) communications in the 30-300 GHz bands [6]. However, for this, the specific propagation characteristics of $\mathrm{mmW}$ need to be taken into consideration. Due to their vulnerability to shadowing, mmW signals can rapidly change with small variation in user environment. Therefore, CA may need to be combined with macrodiversity schemes (e.g., simultaneous connectivity to multiple base stations) to improve communication reliability.

Thanks to the smart device revolution, modern devices have enhanced hardware and also support for multiple network interfaces such as cellular and WiFi, which means they can use different protocols and operate over much wider bandwidths in different spectrum bands. This has created new opportunities and challenges to design enhanced $\mathrm{CA} / \mathrm{CB}$ schemes that can be employed by a unified network across different types of spectrum for increased capacity. Broadly speaking, wireless spectrum can be classified into three different types: 1) licensed spectrum, in which a specific range of frequencies are for exclusive use in a defined geographic area; 2) unlicensed spectrum, in which a specific range of frequencies (such as $2.4 \mathrm{GHz}$ and $5 \mathrm{GHz}$ bands) allow license-exempt operation and are often available worldwide. Such spectrum use is subject to certain limitations, such as limits on power emissions; and 3) shared Access (SA) spectrum, in which in a defined geographic area, a specific range of frequencies (such as $2.3 \mathrm{GHz}$ and TV bands) are allocated to one or more incumbent users, and additional users are allowed to use the spectrum in accordance with sharing rules. SA spectrum can be further subdivided into two types: a) opportunistic access (OA) spectrum (such as TV white spaces) in which a user can use the spectrum as long as she finds it free or she does not cause interference to the incumbent; and b) authorized shared access (ASA) spectrum (such as in the $2.3 \mathrm{GHz}$ band) in which a secondary user requires specific authorization by the 
incumbent.

Recent tutorial articles and surveys have focused on radio resource management schemes for CA in licensed LTE-A networks [3]. However, techniques designed for conventional CA in the licensed bands cannot be directly applied to perform CA in the unlicensed and the SA bands. Unlike in licensed bands, the unpredictability of the interference environment due to uncoordinated competing users in unlicensed bands needs to be considered by a network operator [4]. Also, the number of utilized aggregated carriers needs to be adapted to the changing spectrum environment in SA bands. When multiple networks independently employ $\mathrm{CA} / \mathrm{CB}$, due to the heterogeneous strength of interfering transmissions on overlapping channels, issues such as adjacent-channel interference and channels with dynamic duty cycle patterns become significant. Moreover, not all spectrum bands are equal in terms of coverage or capacity.

Different from the works in the existing literature, in this article we review the recent research efforts relating to the implementations of $\mathrm{CA} / \mathrm{CB}$ techniques that can be used by the operators to offer unified services in licensed, unlicensed, and SA bands. In particular, we identify challenges faced in the scenarios where multiple independent operators decide to combine channels simultaneously in the licensed, unlicensed, and SA bands to obtain wider bandwidth and higher throughput. To this end, we outline directions for future generation wireless networks in this research area. We provide a taxonomy of the use of CA in cellular networks, and highlight differences between the traditional coordinated CA techniques employed in licensed bands and the extension of $\mathrm{CA} / \mathrm{CB}$ techniques to uncoordinated scenarios in the unlicensed and SA bands.

\section{Taxonomy of CA/CB Models in Cellular Networks and the Concept of Anchoring}

Fig. 1 presents a CA/CB taxonomy consisting of two branches: "CA in traditional cellular networks", and "CA/CB: the next generation". Under $\mathrm{CA}$ in traditional cellular networks, the concept of CA was first deployed in high speed packet access (HSPA)-based cellular networks [3]. However, HSPA-based cellular networks support only contiguous intra-band (same band) aggregation of only two carriers. Restrictions in HSPA-based cellular networks (in terms of aggregation of carriers) are relaxed by 3GPP in its LTE-A standard for cellular networks [3]. Unlike HSPA, LTE-A allows coordinated aggregation of contiguous and non-contiguous carriers in both intra and inter-spectrum bands, to achieve high data rate transmissions using up to $100 \mathrm{MHz}$ bandwidth [7]. CA for user terminals in both LTE-A and HSPA cellular networks is performed by central entities (such as base stations) that belong to the same operator. This is efficient 
for these networks as they operate in licensed spectrum, and hence, they have exclusive use of certain bands.

During the last two decades there has been mostly independent technological and market developments in $\mathrm{WiFi}$ and cellular networks. However, the past few years has seen a feverish level of activity in the area of cellular-WiFi integration. Two of the major activities that are ongoing are Hotspot 2.0, under the WiFi Alliance (WFA), and integrated small cell-WiFi networks under the Small Cell Forum [8].

At a high level, the ongoing efforts relating to cellular-WiFi integration under $4 \mathrm{G}$ may be seen as the design of techniques for managing data traffic in mobile operator networks in a smart manner. For example, two such techniques are radio interface offloading and network offloading. In radio interface offloading, traffic is dynamically routed to use the optimal radio interface to suit the particular application and user at hand, taking into account the connectivity cost, network congestion etc. In network offloading, intelligent routing of traffic within the backend networks is performed.

Advances in the next generation of networks will be more than cellular/WiFi integration using interface/network offloading [9]. 5G networks will tie new air interfaces and spectrum together with enhanced versions of LTE-A and WiFi to provide simultaneous and efficient use of multiple radio access technologies. Also, unlike 4G networks, enhanced CA/CB capabilities will provide simultaneous use of licensed, unlicensed and SA bands for increased capacity [9], [10]. In other words, the cellular industry is preparing to adopt newer technologies in which a unified network offers services on licensed, unlicensed and SA bands by employing CA/CB schemes simultaneously in these bands [10], [11]. The need to address the challenges related to the use of enhanced $\mathrm{CA} / \mathrm{CB}$ schemes has led to the techniques which are presented in the second branch of the taxonomy in Fig. 1, and are also the main focus of this article.

In the second branch, "CA/CB: the next generation", three different approaches are presented. In the first approach, aggregation concepts are extended to a unified network offering simultaneous aggregation of carriers across licensed and unlicensed spectrum. From here, there are two broad categories of aggregation schemes: 1) A network using common core and integrated small cells offers the network service (using a technology such as next generation advanced version of LTE-A) on both licensed and unlicensed bands to create fatter data pipes [10]; 2) WiFi is integrated into the operator network, which controls the WiFi interface and decides when and how to simultaneously use the unlicensed spectrum along with the licensed spectrum. In the latter approach, along with CA in licensed bands, CB is used in unlicensed bands. 
bands (such as white the space bands between $50 \mathrm{MHz}$ and $700 \mathrm{MHz}$ ), and across licensed and ASA bands (such as $2.3 \mathrm{GHz}$ band) [12]-[14]. Finally, in the third category, aggregation concepts are extended to both unlicensed and SA bands [10].

To provide the best use of $\mathrm{CA} / \mathrm{CB}$ across all available spectrum resources in the next generation cellular networks, the decision of whether or not to utilize a particular band for $\mathrm{CA} / \mathrm{CB}$, and when to utilize it, needs to be made according to a more complex set of principles than existing CA methods for licensed bands. Operators therefore need a toolbox including different $\mathrm{CA} / \mathrm{CB}$ options so that the right option may be selected for every given scenario. In Fig. 2, we illustrate three methods that can be utilized for the extension of CA/CB in LTE-A, which are further explained in Sections III and IV. For the extension of CA in unlicensed and SA bands using a unified cellular network, licensed spectrum is still of paramount importance, as it provides the foundation for an operator to offer wireless services that is reliable and has predictable performance. Due to this reason, when a network operator employs the extension of CA in the unlicensed and SA bands, it is more appealing that the control signaling always happens on the licensed band, where the quality-of-service (QoS) is ensured. This means no matter what the channel condition in the unlicensed or an SA band is, the crucial signaling information is always communicated properly between the network and device. In [10], this concept is referred to as "anchoring in licensed spectrum".

\section{CA ACRoss LiCEnsed And SA BAnds: Methods And Challenges}

Under this approach, the aggregation of carriers in licensed and SA bands can be utilized for broadband wireless service.

\section{A. Extension of CA in Opportunistic Access (OA) Spectrum Bands}

TV white space spectrum is one of the candidates for OA-based SA. TV white space spectrum refers to the lower frequency bands between $50 \mathrm{MHz}$ and $700 \mathrm{MHz}$. Due to the dynamic nature of white space, the devices in a white space-based network must be aware of its availability. To address this challenge, the extension of CA to white space spectrum can be achieved with an anchor in the licensed spectrum in two different configurations: 1) active scanning and 2) geo-location databases [5], [11], [13].

i) Active scanning: In active scanning of the spectrum, the eNodeB (anchored in the licensed spectrum) decides which of the available channels to aggregate and use. In this approach, the LTE UEs would be sensing the spectrum and sending periodic reports to the eNodeB informing it about spectrum availability. 
The eNodeB would then collate the reports and evaluate whether a change is necessary in the CA configuration, or if the UE ought to continue transmitting and receiving in the same aggregated channels.

ii) Geo-location database: The second deployment model is to have the eNodeB communicating with a geo-location database and allocating/aggregating the available channels to the UEs in its cell. This approach is favored as there is no additional complexity added at the UE or the eNodeB for dynamic radio sensing and evaluation. Here, the LTE UE acts as a Mode I device and the eNodeB acts as a fixed device and communicates with the geo-location database once every 24 hours.

\section{B. Extension of CA in Authorized Shared Access (ASA) Spectrum Bands}

Operators are planning to utilize ASA in bands such as the $2.3 \mathrm{GHz}$ band. Along with macro cells using licensed bands, ASA is well suited for small cell deployment with low power scenarios as the radio propagation is mostly suitable for local area coverage [10]. In any given place and at any given time ASA spectrum is used by either the incumbent user or by the ASA licensee operator. A novel aspect of ASA is that in order to use the ASA bands the operator needs an agreement with the incumbent, based on a sharing framework that outlines the terms of shared use, in which geographical areas to operate, technical conditions for the protection to the incumbent user, and how to vacate the spectrum, if needed. The difference between OA usage and ASA usage is that since the licensees are known and limited in number, both the incumbent and secondary users can be certain of adequate protection.

Most of the existing literature suggest the use of an ASA geo-location database by a network operator, which instructs the relevant base station to start aggregating the ASA channels that are free at a given time. However, the problem of competition among multiple uncoordinated base stations that belong to different operators and can perform CA has not been extensively studied. Next we describe a CA deployment model that is developed in [15] and can be utilized for performing CA in spectrum bands (including ASA bands) where multiple uncoordinated networks compete for the aggregation of carriers with minimized interference.

\section{A Model for Autonomous Aggregation}

We consider that $N$ independent networks operate in the same geographical area and they are notified by a geo-location database that there are $B$ non-overlapping channels available for dynamic usage such as in ASA bands. Each non-overlapping channel has $K_{b}$ carriers in which networks can operate. We consider that each network $n$ can autonomously aggregate a maximum number of $M_{n}$ carriers and all networks 
support both contiguous and non-contiguous CA. Since non-contiguous CA may suffer from out-of-channel (OOC) interference in adjacent frequency channels and also some overhead due to additional guard bands [13], contiguous CA is preferred by a network and we take this into account in our model. When a CA decision is made, the terminals supporting CA between the underlying licensed band (used for control signalling) and the aggregated carriers start operating in the CA mode.

We can model this CA competition as a non-cooperative game denoted by $\mathcal{G}=(\mathcal{N}, \mathcal{A}, r)$. In this game, $\mathcal{N}$ denotes set of players which are the wireless networks. The set of possible actions is denoted by $\mathcal{A}=\left\{\mathcal{A}_{1}, \ldots, \mathcal{A}_{N}\right\}$, where $\mathcal{A}_{n}$ is the set of available actions of the $n^{\text {th }}$ network, and $r$ represents the payoff function. We can define the payoff function in a way that combines the two main aspects of the CA problem: the interference experienced as result of the transmissions of neighboring networks and the cost incurred for inter-band CA. When a network decides to select a set of carriers then its payoff is given as:

$$
r_{n}(\mathbf{a})=\frac{1}{M_{n}} \sum_{c_{n} \in a_{n}}\left(1-\frac{\gamma_{c_{n}}}{\bar{\gamma}_{n}}\right)-\frac{\left(\operatorname{nbands}\left(a_{n}\right)-1\right) \delta}{N_{B_{n}}}
$$

otherwise, its payoff is zero, where $\gamma_{c_{n}}$ is the interference measured in channel $c_{n}$ and normalized in the range of $[0,1]$, and $\bar{\gamma}_{n}$ is the maximum interference that player $n$ can tolerate on a channel (also normalized in $[0,1])$. The function nbands $\left(a_{n}\right)$ returns the number of bands that player $n$ accesses when she selects action $a_{n} \in \mathcal{A}_{\uparrow}$, and $\delta$ is a parameter that each network can tune to reflect its degree of flexibility in performing CA. The game $\mathcal{G}$ is repeated and the networks can change their aggregation decisions, i.e., they change the carriers that they have aggregated.

In our model, each network is not aware of the aggregation decisions of other networks. Each network is able to sense and estimate the level of interference on the available carriers. Each network employs a randomized best response dynamic algorithm [15] that utilizes this sensing/estimation information to reach a stable configuration in which no network has an incentive to unilaterally deviate from its CA selection. In game theoretic terms this is called a Nash Equilibrium (NE). In Fig. 3, we show the performance of the randomized best response dynamic algorithm for autonomous CA in terms of probability of convergence to reach a stable configuration (Nash Equilibrium) under different network scenarios. Fig. 3 shows that by using the randomized best response dynamic algorithm, after only 200 iterations all the studied network scenarios converge to an NE with probability 1. Our results also show that when the number of iterations is less than 200, it is still possible that the network converges to a stable configuration; however, it is not always guaranteed. Figure 3 also shows that more complex scenarios, i.e., having more players 
(autonomous wireless operators) and larger action set (number of carriers that an operator can aggregate), require a higher number of iterations for the network to converge to a stable configuration. For instance, when $N=3, B=4, M=\left[\begin{array}{lll}4 & 4 & 4\end{array}\right], K=\left[\begin{array}{lll}3 & 3 & 3\end{array}\right]$, it takes 200 iterations for the network to converge to a stable configuration whereas when $N=3, B=3, M=\left[\begin{array}{lll}2 & 2 & 2\end{array}\right], K=\left[\begin{array}{lll}2 & 2 & 2\end{array}\right]$ it takes only 100 iterations for the network to converge.

TABLE I

A SUMMARY OF CHALLENGES IN THE DESIGN OF CA/CB TECHNIQUES IN SA BANDS

\begin{tabular}{|c|c|}
\hline Challenges in SA bands & Reasons \\
\hline Inter-carrier load balancing (ICLB) issues & $\begin{array}{l}\text { Aggregated carriers from licensed and SA bands can have } \\
\text { different bandwidths and propagation conditions which in turn } \\
\text { can yield different capacities and can give rise to ICLB issues. }\end{array}$ \\
\hline Autonomous guard band assignment algorithms & $\begin{array}{l}\text { Use of fixed guard bands to mitigate adjacent channel inter- } \\
\text { ference is no longer relevant in CA scenarios where multiple } \\
\text { operators independently perform CA decisions. }\end{array}$ \\
\hline Reliable availability of opportunistically aggregated spectrum & $\begin{array}{l}\text { Availability of a particular CA configuration is not always } \\
\text { guaranteed (as the incumbent may return to a channel). }\end{array}$ \\
\hline Different physical properties of various channels & $\begin{array}{l}\text { White space spectrum (such as in TV bands) has excellent } \\
\text { propagation characteristics. On the other hand, the SA spec- } \\
\text { trum under ASA models targets higher bands where radio } \\
\text { propagation is mostly suitable for local area coverage. }\end{array}$ \\
\hline Independent non-correlated incumbent activity on different channels & $\begin{array}{l}\text { Non-correlated incumbents transmit at different times on dif- } \\
\text { ferent channels. When such channels are aggregated they can } \\
\text { reduce the total channel utilization time. }\end{array}$ \\
\hline Dynamic CA techniques & $\begin{array}{l}\text { As the number of free channels and also the number of } \\
\text { contending users can change with time, this requires dynamic } \\
\text { CA techniques that take into account such network features. }\end{array}$ \\
\hline Reliable control & $\begin{array}{l}\text { SA can be unreliable with unpredictable performance. Hence, } \\
\text { to inform the user nodes about change in spectrum utilization, } \\
\text { reliable control is required. }\end{array}$ \\
\hline Physical layer constraints & $\begin{array}{l}\text { For instance, if the same transmit power is utilized over twice } \\
\text { as many (aggregated) channels, the data rate can double, but } \\
\text { the range can reduce. }\end{array}$ \\
\hline
\end{tabular}

\section{Challenges in the SA Bands}

Much of the existing literature concentrate on designing methods for aggregation of carriers for coordinated network scenarios operating in licensed spectrum bands. However, under the extension of aggregation of channels in SA bands the problem of $\mathrm{CA} / \mathrm{CB}$ has been explored very little. The available spectrum in SA bands at different frequency ranges can be heavily fragmented and without novel aggregating/bonding techniques the secondary devices in SA systems cannot efficiently make use of available spectrum. In Table I, we list some of the challenges in combining channels in the SA spectrum ranges when uncoordinated users are present. 


\section{CA/CB ACRoss Licensed And UnLiCensed Bands: Methods And Challenges}

The bandwidth and number of non-overlapping channels available are key factors when it comes to determining the effectiveness of autonomous $\mathrm{CA} / \mathrm{CB}$ schemes for the aggregation of carriers in licensed and unlicensed spectrum. For instance, in the unlicensed band at $2.4 \mathrm{GHz}$, only three non-overlapping 20 $\mathrm{MHz}$ channels are available, whereas the $5 \mathrm{GHz}$ band has up to $500 \mathrm{MHz}$ of spectrum with more than 20 non-overlapping channels of $20 \mathrm{MHz}$ bandwidth. Moreover, in the $5 \mathrm{GHz}$ band, a network also does not share the spectrum with widely used devices that utilize Bluetooth and cordless phone technology and also equipment such as microwave ovens. This implies less interference.

\section{A. Licensed Plus Unlicensed Using Small Cells}

In this scenario, a heterogeneous network operator deploys small cells in unlicensed bands that use the modified version of the same technology (such as LTE-A or its future versions) that is used in the macro cell (which operates on a licensed band) to co-exist with WiFi systems [10]. In principle LTE-A offers a good opportunity for utilizing $\mathrm{CA}$ in both $2.4 \mathrm{GHz}$ and $5 \mathrm{GHz}$ bands due to its ability to utilize flexible bandwidths of different sizes for component carriers (CCs) (such as 1.4, 3, 5, 10, 15 or $20 \mathrm{MHz}$ ). The main idea behind this scheme is that when suitable carrier(s) are available in the unlicensed band, then a user's primary component carrier (PCC) in the licensed band is aggregated with these secondary component carriers (SCCs) in the unlicensed band. Control signalling (such as ACK/NACK and channel quality indicator [CQI]) is carried on the PCC. A listen-before-talk scheme is utilized before transmission on aggregated channels in the unlicensed aggregated carriers so that the interference with WiFi systems is avoided. In addition, the unlicensed spectrum is used on an "on demand" basis, meaning that only the small cells that have active users are able to transmit in the unlicensed band, and do not transmit at all at other times. This is possible because of a mandatory "anchor" in the licensed band.

There are two main deployment modes for aggregating unlicensed spectrum: 1) supplemental downlink (SDL) mode and 2) time-division duplex (TDD) mode.

1) SDL mode: In the SDL mode, unlicensed spectrum will only be aggregated for the downlink, so that the data rates and capacity are greatly increased in the downlink, keeping the uplink as is. This can be beneficial, especially to address the typically heavy traffic in the downlink.

2) TDD mode: In the TDD mode, the unlicensed spectrum will be aggregated for both downlink and uplink, just like a typical LTE TDD system, and it works like a typical LTE TDD carrier aggregation. 
The advantage here is the flexibility to adjust the amount of resources between the uplink and downlink.

When $\mathrm{CA}$ is utilized in the $5 \mathrm{GHz}$ band, to protect radars using the $5 \mathrm{GHz}$ spectrum, it is also a mandatory regulatory requirement to use Dynamic Frequency Selection and Transmit Power Control (DFS/TPC).

\section{B. Licensed Plus Unlicensed Using WiFi}

Currently, the way WiFi is utilized is suboptimal in the sense that the decision to utilize WiFi for communication and also the decision to whether or not utilize $\mathrm{CB}$ is made independent of real-time information about all the available resources (such as available licensed and unlicensed channels). To overcome this problem and to achieve maximum gain from the efficient utilization of both licensed and unlicensed spectrum bands, CA across the licensed and unlicensed band (using WiFi) is controlled by the network operator. In this approach when CA is utilized across an unlicensed band, then a user's PCC is always in the licensed band. When the operator finds a good radio link using WiFi, then it is the network operator that decides on channel selection/bonding decision for secondary carriers. In the following, we describe the existing $\mathrm{CB}$ methods for secondary carriers.

Bonding in the $2.4 \mathrm{GHz}$ ISM bands: $\mathrm{CB}$ of $40 \mathrm{MHz}$ in $2.4 \mathrm{GHz}$ brings real challenges in terms of both collision avoidance and fairness. Due to the presence of uncoordinated users, maintaining accurate physical carrier sensing and virtual carrier sensing on two $20 \mathrm{MHz}$ subchannels in parallel incurs additional overhead costs. To address this challenge, in $40 \mathrm{MHz} \mathrm{CB}$, a primary $20 \mathrm{MHz}$ channel and a secondary 20 $\mathrm{MHz}$ channel are defined. When a user wants to transmit using a $40 \mathrm{MHz}$ band, it performs usual carrier sensing on the primary channel and it performs a carrier sensing for a shorter duration on the secondary channel to make sure it is clear too. If clear, the packet is transmitted using a $40 \mathrm{MHz}$ band; otherwise, the device can either: 1) transmit in the primary $20 \mathrm{MHz}$ channel; or 2) back off again, and then recheck if the full $40 \mathrm{MHz}$ bandwidth is available [5].

Bonding in the $5 \mathrm{GHz}$ ISM bands: Using 802.11ac, up to eight $20 \mathrm{MHz}$ contiguous channels can be bonded. It is required in the $802.11 \mathrm{ac}$ standard that a cellular network bonding $40 \mathrm{MHz}$ (or $80 \mathrm{MHz}$ and so on) should also allow 802.11a or 802.11n clients to associate. For instance, there can be nearby other uncoordinated APs that use 802.11a or 802.11n. This scenario leads to the nodes having different virtual carrier sensing, hence they can transmit at different times on the different subchannels, this can lead to different overlapping transmission times on bonded channels. Currently, IEEE 802.11ac defines an enhanced RTS/CTS protocol for CB of multiple channels by uncoordinated users [5]. To ensure fairness 
among heterogeneous $\mathrm{CB}$ users, a network that is using $\mathrm{CA} / \mathrm{CB}$ across licensed and unlicensed bands needs to determine not only when unlicensed channels are available for CB but also how many are interference free around both the initiator and the responder nodes (see examples in Fig. 4). It can be seen in Fig. 4 that in this enhanced protocol, $\mathrm{CB}$ is allowed only on those channels which are found interference-free by both the initiator and the recipient of communication.

\section{Challenges in the Unlicensed Bands}

It is worth noting that the increased complexity brought about by the addition of aggregation opportunities in unlicensed bands require more efficient $\mathrm{CA} / \mathrm{CB}$ techniques. In Table II, we list some of the challenges faced by a network operator in combining channels in the unlicensed spectrum ranges when uncoordinated users are present.

TABLE II

A SUMMARY OF CHALLENGES IN THE DESIGN OF CA/CB TECHNIQUES IN THE UNLICENSED BANDS

\begin{tabular}{|c|l|}
\hline Challenges in unlicensed bands & \multicolumn{1}{|c|}{ Reasons } \\
\hline \hline Dynamic frequency selection (DFS) & $\begin{array}{l}\text { DFS in the 5 GHz band should avoid incumbents such as } \\
\text { domain weather radar (if present) }\end{array}$ \\
\hline Multiple uncoordinated nearby APs & $\begin{array}{l}\text { When using CB if the same transmit power is utilized over } \\
\text { twice as many (bonded) channels, the data rate doubles, but } \\
\text { the range can reduce. }\end{array}$ \\
\hline CB decision using RSSI & $\begin{array}{l}\text { Need to avoid multiple uncoordinated nearby APs over bonded } \\
\text { channels. Due to uncoordinated APs, a single overlapping } \\
\text { channel can affect the wider bandwidth of this AP. }\end{array}$ \\
\hline Selection of modulation and coding scheme (MCS) along with rate \\
adaptation & $\begin{array}{l}\text { Recent measurements in [4] show that if RSSI is above the } \\
\text { minimum input sensitivity of a bonded channel, then a bonded } \\
\text { channel always performs better than a single channel given that } \\
\text { interference is not very high. }\end{array}$ \\
\hline Channel leakage due to neighboring user transmissions & $\begin{array}{l}\text { Proper selection of MCS along with rate adaptation can } \\
\text { maximize the benefits of CB. }\end{array}$ \\
\hline Knowlith strong signal strengths should bond orthogonal channels. \\
withe of the physical rates of competing links & $\begin{array}{l}\text { Knowing the physical rates of competing links, i.e., the links } \\
\text { that are in carrier sensing range, helps in making a good } \\
\text { bonding decision. }\end{array}$ \\
\hline
\end{tabular}

\section{CONCLUSiON}

Carrier aggregation/channel bonding (CA/CB) between licensed, unlicensed, and shared access (SA) carriers is an innovation in the next generation cellular networks. This has the potential to offer increased user data rates, while enabling clients to co-exist efficiently with existing cellular systems. In this article, we have discussed different techniques where $\mathrm{CA} / \mathrm{CB}$ is performed between licensed, unlicensed, and SA carriers. Despite the similarity that (for reliability) almost all the CA methods in different bands need a licensed PCC to control secondary carriers, there are unique challenges to CA due to the different types 
of spectrum utilization regimes. For instance, due to multiple overlapping WiFi channels and also several other uncoordinated users using frequency bands of different sizes (for example the 802.15.4 uses $2 \mathrm{MHz}$ ), efficient CA between licensed carrier and unlicensed carriers (in the $2.4 \mathrm{GHz}$ band) can be challenging. On the other hand, the $2.4 \mathrm{GHz}$ band is very close to the $2.5 \mathrm{GHz}$ LTE band. This implies that modification of the RF circuits will be easier in devices to aggregate carriers in the $2.4 \mathrm{GHz}$ band using the LTE air interface. $\mathrm{CA} / \mathrm{CB}$ between licensed and unlicensed carriers in the $5 \mathrm{GHz}$ band provides more opportunities due to the availability of many non-overlapping channels; however, to efficiently utilize wider bandwidths, a user needs to avoid this bandwidth to overlap with other uncoordinated users, all potentially on different channels. This is due to the reason that multiple uncoordinated media access control (MAC) over a single overlapped channel can affect the wider bandwidth of this user.

In the scenarios where $\mathrm{CA} / \mathrm{CB}$ is performed between licensed and $\mathrm{SA}$ carriers, it is important to aggregate those carriers that are more reliable in terms of availability. This is due to the reason that at a given time instant, SA bands are mostly fragmented and the availability of a particular CA/CB configuration is not always guaranteed. Taking these different challenges into account, due to the dynamic nature of unlicensed and SA carriers, and also due to the presence of uncoordinated users in these carriers, careful design of new $\mathrm{CA} / \mathrm{CB}$ techniques is required for the next generation cellular wireless networks.

\section{REFERENCES}

[1] C. Wang, F. Haider, X. Gao, X. You, Y. Yang, D. Yuan, H. Aggoune, H. Haas, S. Fletcher, and E. Hepsaydir, "Cellular architecture and key technologies for 5G wireless communication networks," IEEE Communications Magazine, vol. 52, no. 2, pp. 122-130, 2014.

[2] F. Boccardi, R. W. Heath, A. Lozano, T. L. Marzetta, and P. Popovski, "Five disruptive technology directions for 5G," IEEE Communications Magazine, vol. 52, no. 2, pp. 74-80, 2014.

[3] H. Lee, S. Vahid, and K. Moessner, "A survey of radio resource management for spectrum aggregation in LTE-advanced," IEEE Communications Surveys Tutorials, vol. 16, no. 2, pp. 745-760, 2014.

[4] L. Deek, E. G. Villegas, E. Belding, S. Lee, and K. Almeroth, "Intelligent channel bonding in 802.11n WLANs," IEEE Transactions on Mobile Computing, vol. 13, no. 6, pp. 1242-1255, 2014.

[5] Cisco. (2014) 802.11ac: The fifth generation of wi-fi. [Online]. Available: http://www.cisco.com/c/en/us/products/collateral/wireless/ aironet-3600-series/white_paper_c11-713103.pdf

[6] S. Rangan, T. Rappaport, and E. Erkip, "Millimeter-wave cellular wireless networks: Potentials and challenges," Proceedings of the IEEE, vol. 102, no. 3, pp. 366-385, March 2014.

[7] K. Pedersen, F. Frederiksen, C. Rosa, H. Nguyen, L. Garcia, and Y. Wang, "Carrier aggregation for LTE-advanced: Functionality and performance aspects," IEEE Communications Magazine, vol. 49, no. 6, pp. 89-95, 2011.

[8] InterDigital. (2012) Cellular-WiFi integration. [Online]. Available: http://www.interdigital.com/wp-content/uploads/2012/08/Cellular_ WiFi_Integration-White-Paper.pdf 
[9] J. Andrews, S. Buzzi, W. Choi, S. Hanly, A. Lozano, A. Soong, and J. Zhang, "What will 5G be?" IEEE Journal on Selected Areas in Communications, vol. 32, no. 6, pp. 1065-1082, June 2014.

[10] Qualcomm. (2013) 1000x: More spectrum-especially for small cells. [Online]. Available: http://www.qualcomm.com/media/documents/ 1000x-more-spectrum-especially-small-cells

[11] P. Surampudi and S. Mohanty. (2011) LTE-advanced in white space: A complementary technology. [Online]. Available: http://go.radisys.com/rs/radisys/images/paper-lte-white-space.pdf

[12] J. Xiao, R. Hu, Y. Qian, L. Gong, and B. Wang, "Expanding LTE network spectrum with cognitive radios: From concept to implementation,” IEEE Wireless Communications, vol. 20, no. 2, pp. 12-19, 2013.

[13] H. A. B. Salameh, M. Krunz, and D. Manzi, "Spectrum bonding and aggregation with guard-band awareness in cognitive radio networks," IEEE Transactions on Mobile Computing, vol. 13, no. 3, pp. 569-581, March 2014.

[14] S. Joshi, P. Pawelczak, D. Cabric, and J. Villasenor, "When channel bonding is beneficial for opportunistic spectrum access networks," IEEE Transactions on Wireless Communications, vol. 11, no. 11, pp. 3942-3956, November 2012.

[15] H. Ahmadi, I. Macaluso, and L. A. DaSilva, "Carrier aggregation as a repeated game: Learning algorithms for efficient convergence to a nash equilibrium," in Proceedings of IEEE Globecom, Dec 2013, pp. 1233-1239.

Zaheer Khan is a research fellow at Centre for Wireless Communications (CWC), University of Oulu, Finland since 2012. He obtained his Master from Högskolan i Borås, Sweden in 2007, and his Ph.D. from University of Oulu, Finland, in 2011. He is the recipient of the Marie Curie fellowship for the year 2007-2008 and Academy of Finland Postdoctoral Researcher Award for the year 2013-2016. His research interests include application of game theory to model distributed wireless networks, cognitive and cooperative communications, and wireless social networks.

Hamed Ahmadi received his M.Sc. degree in software engineering from National Aerospace University of Kharkiv (KhAI), Kharkiv, Ukraine, in 2008, and Ph.D. in electrical engineering from National University of Singapore, 2012. He was with the R\&D department of Zarrin Afzar electric company, Mashhad, Iran, from 2004 to 2006. He is currently a research fellow at CTVR, the telecommunications research center, Trinity College Dublin. His research interest includes Machine learning, game theory, Cognitive Radio, dynamic spectrum allocation, radio resource allocation, small-cell and self-organizing networks. 
Ekram Hossain (S'98-M'01-SM'06) is a Professor (since March 2010) in the Department of Electrical and Computer Engineering at University of Manitoba, Winnipeg, Canada. He received his Ph.D. in Electrical Engineering from University of Victoria, Canada, in 2001. Dr. Hossain's current research interests include design, analysis, and optimization of wireless/mobile communications networks, cognitive radio systems, and network economics. He has authored/edited several books in these areas (http://home.cc.umanitoba.ca/ hossaina). Dr. Hossain serves as the Editor-in-Chief for the IEEE Communications Surveys and Tutorials. Also, he is a member of the IEEE Press Editorial Board. Dr. Hossain has won several research awards including the University of Manitoba Merit Award in 2010 and 2014 (for Research and Scholarly Activities). He is a Distinguished Lecturer of the IEEE Communications Society (2012-2015).

Marceau Coupechoux is an Associate Professor at Telecom ParisTech since 2005. He obtained his Master from Telecom ParisTech in 1999 and from University of Stuttgart in 2000, and his Ph.D. from Institut Eurecom, Sophia-Antipolis, France, in 2004. From 2000 to 2005, he was with Alcatel-Lucent. In the Computer and Network Science department of Telecom ParisTech, he is currently working on cellular networks, wireless networks, ad hoc networks, cognitive networks, focusing mainly on layer 2 protocols, scheduling and resource management.

Luiz DaSilva (SM) holds the Stokes Professorship in Telecommunications in the department of Electronic and Electrical Engineering at Trinity College Dublin. He is also a Professor in the Bradley Department of Electrical and Computer Engineering at Virginia Tech. Prof. DaSilva's research focuses on distributed and adaptive resource management in wireless networks, and in particular cognitive radio networks and the application of game theory to wireless networks. He is currently a principal investigator on research projects funded by the National Science Foundation, the Science Foundation Ireland, and the European Commission under Framework Programme 7.

Janne J . Lehtomäki Janne J . Lehtomäki graduated with an M. Sc. and Ph. D. in Telecommunications from the University of Oulu, Finland, in 1999 and 2005, respectively. Currently, he is a senior research fellow at the University of Oulu, Centre for Wireless Communications (CWC). His research interests are in nanonetworks, spectrum measurements, energy detection, and cognitive radio networks. He co-authored the paper receiving the Best Paper Award in IEEE WCNC 2012. 


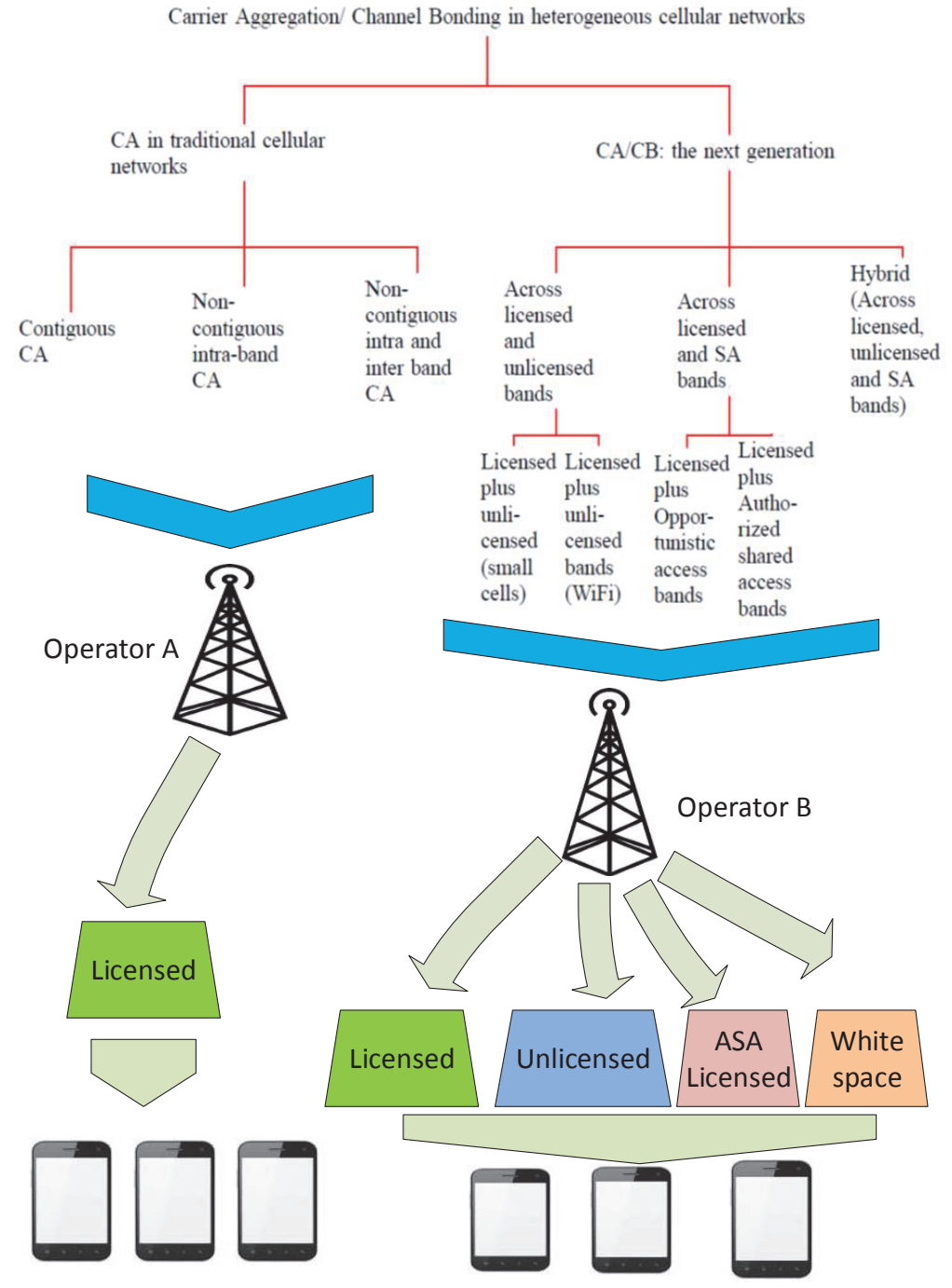

Fig. 1. CA/CB taxonomy and different spectrum band utilization for aggregation in traditional and next generation networks. 


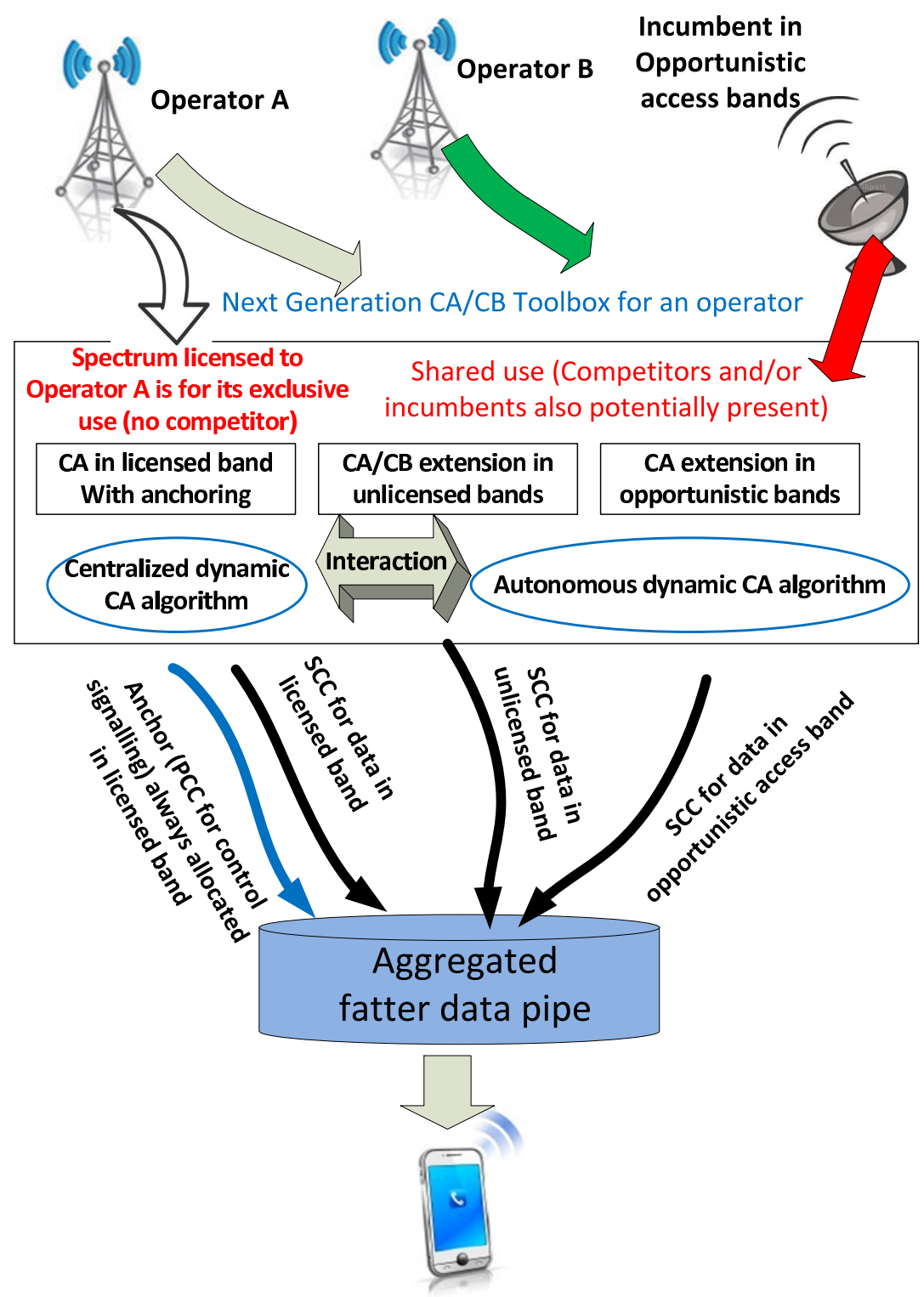

Fig. 2. Next generation CA/CB toolbox. PCC denotes primary component carrier and SCC denotes secondary component carrier. 


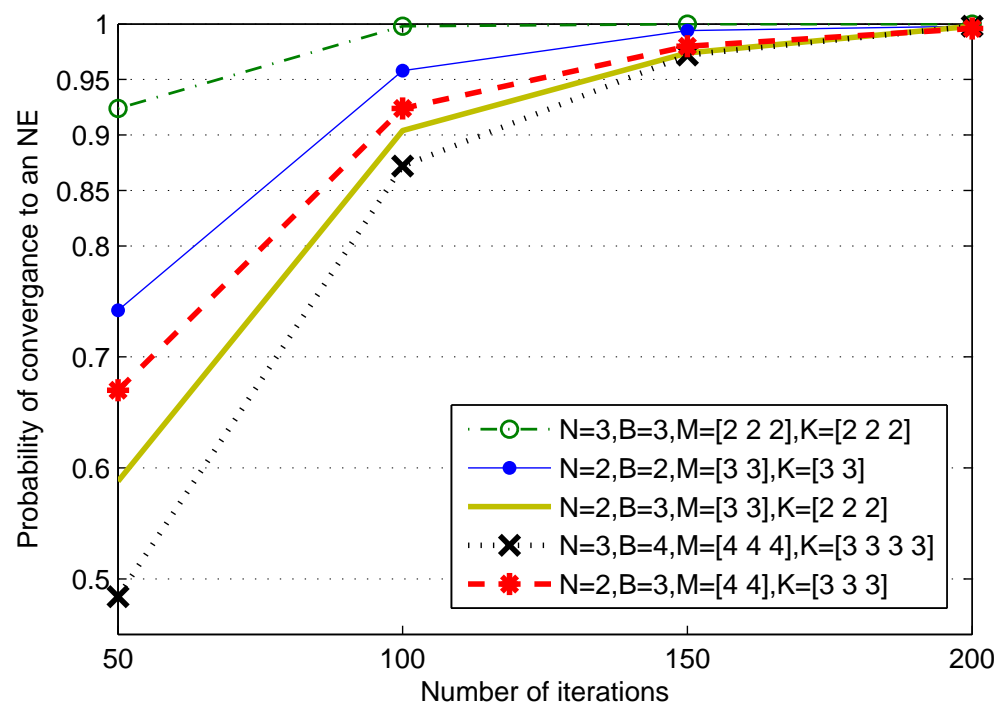

Fig. 3. Convergence probability of the randomized best response dynamic to an NE for different scenarios. $K$ shows the number of carriers in each band and $M$ shows the number of carriers that each user can aggregate. 
RTS= Request to send, $\mathrm{CTS}=$ Clear to send, $\mathrm{ACK}=$ Acknowledgement

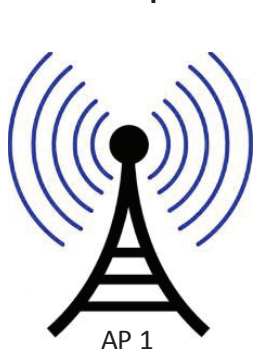

AP 1 bonds four $20 \mathrm{MHz}$ [total $80 \mathrm{MHz}$ ] channels to communicate in no interference scenario

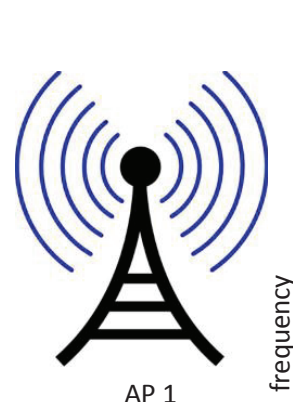

AP 1

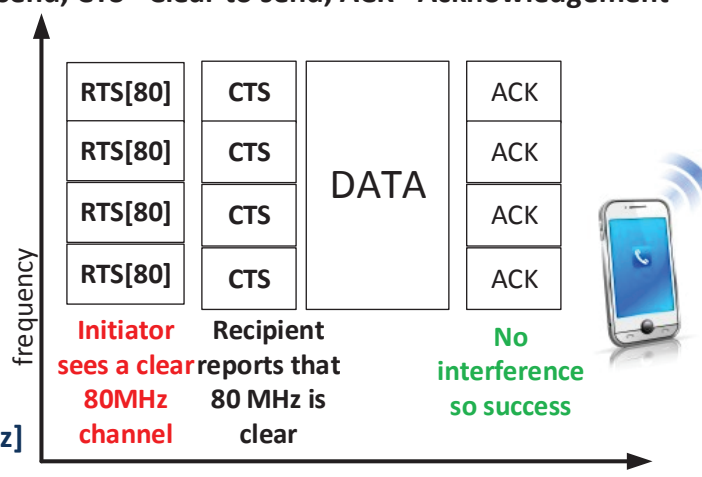

a)
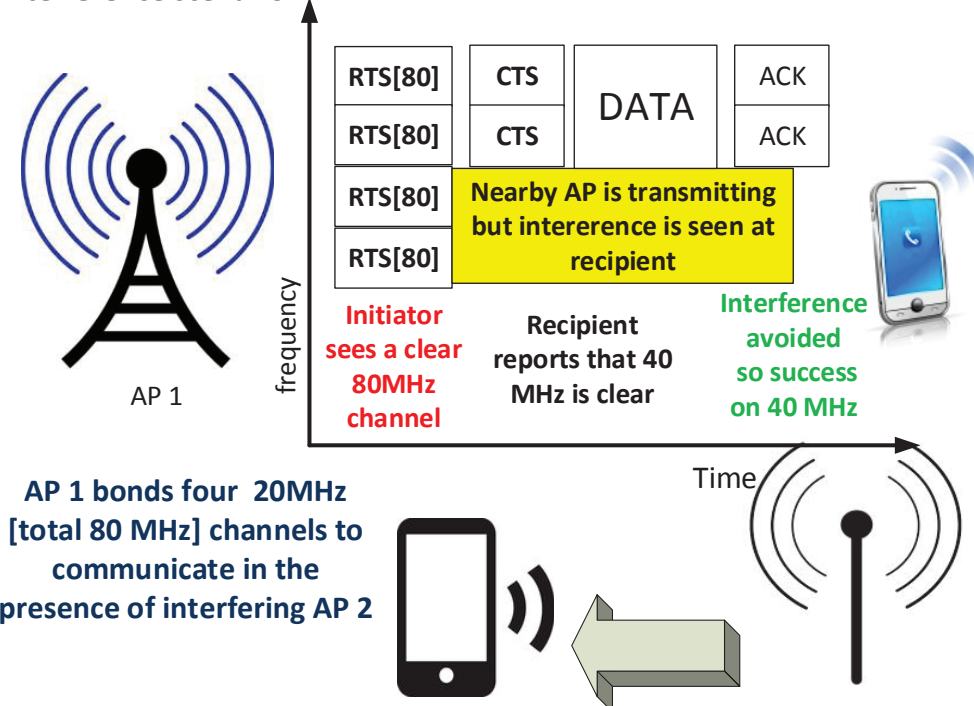

b)

AP 2

Fig. 4. Examples of enhanced RTS/CTS channel bonding protocol: a) When access point (AP) 1 is bonding to communicate in the absence of interference; and b) When AP 1 is bonding to communicate in the presence of interference. 